PROCEEDINGS OF THE

AMERICAN MATHEMATICAL SOCIETY

Volume 134, Number 2, Pages 509-519

S 0002-9939(05)08187-6

Article electronically published on August 12, 2005

\title{
A PROBLEM OF BAERNSTEIN ON THE EQUALITY OF THE $p$-HARMONIC MEASURE OF A SET AND ITS CLOSURE
}

\author{
ANDERS BJÖRN, JANA BJÖRN, AND NAGESWARI SHANMUGALINGAM
}

(Communicated by Andreas Seeger)

\begin{abstract}
A. Baernstein II (Comparison of p-harmonic measures of subsets of the unit circle, St. Petersburg Math. J. 9 (1998), 543-551, p. 548), posed the following question: If $G$ is a union of $m$ open arcs on the boundary of the unit disc $\mathbf{D}$, then is $\omega_{a, p}(G)=\omega_{a, p}(\bar{G})$, where $\omega_{a, p}$ denotes the $p$-harmonic measure? (Strictly speaking he stated this question for the case $m=2$.) For $p=2$ the positive answer to this question is well known. Recall that for $p \neq 2$ the $p$-harmonic measure, being a nonlinear analogue of the harmonic measure, is not a measure in the usual sense.

The purpose of this note is to answer a more general version of Baernstein's question in the affirmative when $1<p<2$. In the proof, using a deep trace result of Jonsson and Wallin, it is first shown that the characteristic function $\chi_{G}$ is the restriction to $\partial \mathbf{D}$ of a Sobolev function from $W^{1, p}(\mathbf{C})$.

For $p \geq 2$ it is no longer true that $\chi_{G}$ belongs to the trace class. Nevertheless, we are able to show equality for the case $m=1$ of one arc for all $1<p<\infty$, using a very elementary argument. A similar argument is used to obtain a result for starshaped domains.

Finally we show that in a certain sense the equality holds for almost all relatively open sets.
\end{abstract}

\section{INTRODUCTION}

Baernstein [1], p. 548, posed the following question: If $G \subset \mathbf{D}$ is a union of $m$ open arcs, then is $\omega_{a, p}(G)=\omega_{a, p}(\bar{G})$ ? Here $\omega_{a, p}$ denotes the $p$-harmonic measure. (Strictly speaking he stated this question for the case $m=2$.) For the linear case $p=2$ the positive answer to this question is well known. Recall that for $p \neq 2$ the $p$-harmonic measure, being a nonlinear analogue of the harmonic measure, is not a measure in the usual sense.

We answer Baernstein's question in the following proposition.

Received by the editors September 27, 2004.

2000 Mathematics Subject Classification. Primary 31C45; Secondary 30C85, 31A25, 31B20, 31C15, 46E35.

Key words and phrases. Ahlfors regular, Dirichlet problem, $d$-set, Lipschitz domain, Minkowski dimension, $p$-harmonic function, $p$-harmonic measure, Sobolev function, starshaped, trace, unit disc.

We thank Juha Heinonen for drawing our attention to the question of Baernstein.

The first two authors were supported by the Swedish Research Council and Gustaf Sigurd Magnuson's fund of the Royal Swedish Academy of Sciences. The second author did this research while she was at Lund University.

The third author was partly supported by NSF grant DMS 0243355.

(C)2005 American Mathematical Society Reverts to public domain 28 years from publication 
Proposition 1.1. Let $1<p<\infty$ and let $\Omega=\mathbf{D}$ be the unit disc in the complex plane. Let $G \subset \partial \mathbf{D}$ be the union of $m$ open arcs. If $m=1$ or $p \leq 2$, then

$$
\omega_{a, p}(G)=\omega_{a, p}(\bar{G}) \quad \text { for all } a \in \mathbf{D} .
$$

As far as we know, Baernstein's question remains open if $m>1$ and $p>2$.

In the proof for $1<p<2$, it is first shown that the characteristic function $\chi_{G}$ is the restriction to $\partial \mathbf{D}$ of a $p$-quasicontinuous Sobolev function from $W^{1, p}(\mathbf{C})$, using a deep trace result of Jonsson and Wallin (some earlier results would also suffice for the unit disc). This, together with a recent result of the authors of this note [3], Corollary 5.2, demonstrates the equality in question.

For $p \geq 2$ it is no longer true that $\chi_{G}$ belongs to the trace class, and the above outlined proof breaks down. Nevertheless, we are able to show equality for the case $m=1$ (a single arc) for all $1<p<\infty$, using a very elementary argument. In Proposition 3.1 we obtain a similar result for starshaped domains $\Omega$ and a starshaped set on its boundary.

In the literature there are, of course, invariance results for the $p$-harmonic measure. Kurki [1], Theorem 1.1 (or Björn-Björn-Shanmugalingam [3], Corollary 7.5), states that if $K$ is a compact set and $C_{p}(E)=0$, then $\bar{\omega}_{a, p}(K)=\bar{\omega}_{a, p}(K \cup E)$. As far as we are aware, Kurki's result (together with its dual version) contains all other invariance results in the literature for $p \neq 2$. Unfortunately, as Baernstein points out, Kurki's result cannot be applied to our problem.

For bounded domains $\Omega \subset \mathbf{R}^{n}$ and $E \subset \partial \Omega$, we use the full power of JonssonWallin's result to give conditions on $\Omega$ and $E$ such that $\omega_{a, p}\left(\operatorname{int}_{\partial \Omega} E\right)=\omega_{a, p}(E)=$ $\omega_{a, p}(\bar{E})$. For the full statement of the main result we refer to Section 4 but here we give a corollary.

Proposition 1.2. Let $1<p<n$ and let $\Omega \subset \mathbf{R}^{n}$ be a bounded Lipschitz domain. Moreover, let $E \subset \partial \Omega$ be such that $\operatorname{dim}_{M} \partial_{\partial \Omega} E<n-p$. Then

$$
\omega_{a, p}\left(\operatorname{int}_{\partial \Omega} E\right)=\omega_{a, p}(E)=\omega_{a, p}(\bar{E}) \quad \text { for all } a \in \Omega .
$$

Here int ${ }_{\partial \Omega} E$ and $\partial_{\partial \Omega} E$ are the interior and the boundary, respectively, of $E$ in the induced subset topology on $\partial \Omega$. The upper Minkowski dimension is denoted by $\operatorname{dim}_{M}$; see Federer [7, Section 3.2.37, or Mattila [13, Sections 5.3 and 5.5, for the definition.

If $n=2$ and (1.1) holds for some $a \in \Omega$, then (1.1) holds for all $a \in \Omega$, by Manfredi [12, Theorem 2. Whether this implication holds for $n \geq 3$ is an open problem.

Finally we show in Proposition 3.2 that in a certain sense equality holds for almost all relatively open sets.

It should be pointed out that in Carnot-Carathéodory spaces a result similar to Theorem 4.1 can be obtained by replacing the trace result of Jonsson-Wallin by the trace result of Danielli-Garofalo-Nhieu [5], 6]. For general metric spaces see also Björn-Björn-Shanmugalingam [4].

\section{Notation AND DEFINitions}

We let $W^{1, p}(\Omega)$ be the standard Sobolev space of all functions in $L^{p}(\Omega)$ whose distributional gradients also belong to $L^{p}(\Omega)$. We also define the Sobolev capacity

$$
C_{p}(E)=\inf \int_{\mathbf{R}^{n}}\left(|f|^{p}+|\nabla f|^{p}\right) d x,
$$


where the infimum is taken over all $f \in W^{1, p}\left(\mathbf{R}^{n}\right)$ such that $f=1$ in an open set containing $E$.

A function $u \in W_{\mathrm{loc}}^{1, p}(\Omega)$ is $p$-harmonic if it is continuous and a weak solution of the equation

$$
\Delta_{p} u:=\operatorname{div}\left(|D u|^{p-2} D u\right)=0 \quad \text { in } \Omega
$$

it is $p$-superharmonic if it is lower semicontinuous and

$$
\Delta_{p} u \leq 0 \quad \text { in } \Omega
$$

in the distributional sense.

We define the upper Perron solution of $f$ by letting

$$
\bar{P} f(x)=\inf u(x), \quad x \in \Omega,
$$

where the infimum is taken over all $p$-superharmonic functions $u$ on $\Omega$ bounded below and such that

$$
\liminf _{\Omega \ni y \rightarrow x} u(y) \geq f(x) \text { for all } x \in \partial \Omega .
$$

The lower Perron solution can be defined in a dual fashion, or simply by letting

$$
\underline{P} f(x)=-\bar{P}(-f)(x) \text {. }
$$

If $\bar{P} f=\underline{P} f$, then we denote the common function by $P f$, and $f$ is said to be resolutive. It is always true that $\underline{P} f \leq \bar{P} f$. Apart from the case when $\bar{P} f \equiv \pm \infty$, $\bar{P} f$ is a $p$-harmonic function.

It should be observed that if $f \in C(\partial \Omega)$, then $f$ is resolutive; see, e.g., HeinonenKilpeläinen-Martio 9, Theorem 9.25, or Björn-Björn-Shanmugalingam [3, Theorem 6.1. In [3], Corollary 5.2, it was shown that if $f$ is a $p$-quasicontinuous representative of an equivalence class in $W^{1, p}\left(\mathbf{R}^{n}\right)$, then $f$ is resolutive; this will be essential to us. A function $f$ is $p$-quasicontinuous if for every $\varepsilon>0$ there is a set $E$ with $C_{p}(E)<\varepsilon$ such that $\left.f\right|_{\mathbf{R}^{n} \backslash E}$ is continuous.

The upper and lower $p$-harmonic measures of $E \subset \partial \Omega$ evaluated at $a \in \Omega$ are defined by

$$
\bar{\omega}_{a, p}(E ; \Omega)=\bar{\omega}_{a, p}(E)=\bar{P} \chi_{E}(a) \quad \text { and } \quad \underline{\omega}_{a, p}(E ; \Omega)=\underline{\omega}_{a, p}(E)=\underline{P} \chi_{E}(a) .
$$

When $\underline{\omega}_{a, p}(E)=\bar{\omega}_{a, p}(E)$ we denote the common value by $\omega_{a, p}(E ; \Omega)=\omega_{a, p}(E)$.

It should be observed that due to the nonlinearity of $\Delta_{p}$, the $p$-harmonic measure is not a measure when $p \neq 2$. For more on $p$-harmonic measures and their history, see [9], Chapter 11. (Let us also refer the interested reader to the results in Section 8 in Björn-Björn-Shanmugalingam [2] and Corollary 7.5 in [3].)

Throughout this paper, $C$ denotes a positive constant whose exact value is unimportant, can change even within the same line, and depends only on fixed parameters. The ball with centre $x$ and radius $r$ is denoted by $B(x, r)$.

\section{Elementary Results}

We start with some elementary proofs.

Proof of Proposition 1.1 for the case $m=1$. We use complex notation to simplify some of the expressions. Let $G_{\theta}=\{z \in \partial \mathbf{D}:|\arg z|<\theta\}$. By rotating $\mathbf{D}$ if necessary, we can assume that $G=G_{\delta}$ for some $\delta \in(0, \pi)$. 
Let $u(z)=\omega_{z, p}\left(G_{\delta}\right)$ and $\bar{u}(z)=\omega_{z, p}\left(\bar{G}_{\delta}\right)$. Note that the upper and lower $p$-harmonic measures coincide in this case by Proposition 9.31 in Heinonen-Kilpeläinen-Martio 9] (or Corollary 7.4 in [3]).

For $0<x<1$, let $r(x)=\left|e^{i \delta}-x\right|, \delta(x)=\arg \left(e^{i \delta}-x\right)$, and for $z \in B(x, r(x))$, let

$$
\begin{aligned}
v_{x}(z) & =\omega_{z, p}(\{y \in \partial B(x, r(x)):|\arg (y-x)|<\delta(x)\} ; B(x, r(x))) \\
& =\omega_{(z-x) / r(x), p}\left(G_{\delta(x)}\right) .
\end{aligned}
$$

By the comparison principle in the domain $\mathbf{D} \cap B(x, r(x))$, we see that $v_{x} \leq u$ in $\mathbf{D} \cap B(x, r(x))$. On the other hand, by the comparison principle again, $u(z) \leq$ $\bar{u}(z) \leq \omega_{z, p}\left(G_{\delta(x)}\right)=v_{x}(x+r(x) z)$ for all $z \in \mathbf{D}$ and $0<x<1$. Thus, using the continuity of the $p$-harmonic function $\bar{u}$ and by the fact that every $z \in \mathbf{D}$ belongs to $\mathbf{D} \cap B(x, r(x))$ for sufficiently small $x>0$,

$$
u(z) \leq \bar{u}(z)=\lim _{x \rightarrow 0+} \bar{u}((z-x) / r(x)) \leq \lim _{x \rightarrow 0+} v_{x}(z) \leq u(z) \quad \text { for } z \in \mathbf{D} .
$$

The proof above immediately generalizes to spherical caps on balls in $\mathbf{R}^{n}$.

In the next proposition we apply the method of the above proof to starshaped domains.

Proposition 3.1. Let $1<p<\infty$ and let $\Omega \subset \mathbf{R}^{n}$ be a bounded domain which is starshaped with respect to $0 \in \partial \Omega$. Let $E \subset \partial \Omega$ be starshaped with respect to 0 and such that

$$
\delta \bar{E} \subset E \quad \text { whenever } 0<\delta<1 \text {. }
$$

Then

$$
\bar{\omega}_{a, p}(E)=\bar{\omega}_{a, p}(\bar{E}) \text { and } \underline{\omega}_{a, p}(E)=\underline{\omega}_{a, p}(\bar{E}) \quad \text { for all } a \in \Omega .
$$

If $\Omega$ is a p-regular domain, or $\delta \bar{E} \subset \operatorname{int}_{\partial \Omega} E$ when $0<\delta<1$, then

$$
\omega_{a, p}(E)=\omega_{a, p}(\bar{E}) \quad \text { for all } a \in \Omega \text {. }
$$

For the definition of $p$-regularity see [2, 3] or [9].

The starshapedness of $E$ does not in general guarantee that $\delta \bar{E} \subset E$ for $0<$ $\delta<1$, even if we insist on the starcenter 0 lying in $\operatorname{int}_{\partial \Omega} E$, as exhibited by, e.g., letting $E=\mathbf{D} \backslash\left[\frac{1}{2}, 1\right) \subset \mathbf{C}$ or $E=\mathbf{D} \cup\{z:|z|<2$ and $0<\arg z<\pi / 4\}$. Here we take the ambient space to be $\mathbf{R}^{3}$ and $\Omega$ to be the cube $(-2,2)^{2} \times(0,4)$.

Note that if $\delta \bar{E} \subset \operatorname{int}_{\partial \Omega} E$ when $0<\delta<1$, then $\bar{E}=\overline{\operatorname{int}_{\partial \Omega} E}$, and thus also $\omega_{a, p}\left(\operatorname{int}_{\partial \Omega} E\right)=\omega_{a, p}(\bar{E})$.

This proposition can be applied to the situation when $\Omega \subset \mathbf{R}^{2}$ is a rectangle, and $E \subset \partial \Omega$ is connected, and either lies on one or two of the rectangle's sides or has a complement which lies on one or two sides. In the latter case one needs to use the duality condition $\bar{\omega}_{a, p}(A)=1-\underline{\omega}_{a, p}(\partial \Omega \backslash A)$.

Proof. Let $0<\delta<1$. Then by a rescaling and the comparison principle, we have

$$
\bar{\omega}_{a, p}(\bar{E})=\bar{\omega}_{\delta a, p}(\delta \bar{E} ; \delta \Omega) \leq \bar{\omega}_{\delta a, p}(E) \text { for } a \in \Omega .
$$

Letting $\delta \rightarrow 1-$ and using the continuity of the $p$-harmonic function $a \mapsto \bar{\omega}_{a, p}(E)$ (note that $\lim _{\delta \rightarrow 1-} \delta a=a$ ), we obtain

$$
\bar{\omega}_{a, p}(\bar{E}) \leq \bar{\omega}_{a, p}(E) \leq \bar{\omega}_{a, p}(\bar{E}) \quad \text { whenever } a \in \Omega
$$

The proof for $\underline{\omega}$ is similar. 
If $\Omega$ is a $p$-regular domain, then Proposition 9.31 in Heinonen-KilpeläinenMartio [9] (or Corollary 7.4 in [3]) shows that $\bar{\omega}_{a, p}(\bar{E})=\underline{\omega}_{a, p}(\bar{E})$ from which the conclusion follows.

If $\delta \bar{E} \subset \operatorname{int}_{\partial \Omega} E$ when $0<\delta<1$, we proceed as follows. For $0<\delta<1$, we can find $f_{\delta} \in C(\partial \Omega)$ such that $f_{\delta}=1$ on $\delta \bar{E}$ and $f_{\delta}=0$ on $\partial \Omega \backslash E$. Thus, by the resolutivity of continuous functions, we obtain

$$
\bar{\omega}_{a, p}(\bar{E})=\bar{\omega}_{\delta a, p}(\delta \bar{E} ; \delta \Omega) \leq \bar{\omega}_{\delta a, p}(\delta \bar{E} ; \Omega) \leq P f_{\delta}(\delta a) \leq \underline{\omega}_{\delta a, p}(E) \quad \text { for } a \in \Omega .
$$

Letting $\delta \rightarrow 1$ - and using the continuity of the $p$-harmonic function $a \mapsto \underline{\omega}_{a, p}(E)$ we obtain

$$
\bar{\omega}_{a, p}(\bar{E}) \leq \underline{\omega}_{a, p}(E) \leq \bar{\omega}_{a, p}(\bar{E}) \quad \text { when } a \in \Omega,
$$

which combined with the first part completes the proof.

Proposition 3.2. Let $1<p<\infty$ and let $\Omega \subset \mathbf{R}^{n}$ be a bounded domain. Let $\left\{E_{r}\right\}_{r \in(0,1)}$ be a family of sets such that $\bar{E}_{s} \subset \operatorname{int}_{\partial \Omega} E_{r} \subset \partial \Omega$ if $0<s<r<1$. Then for almost every $r$ (with respect to the Lebesgue measure on the interval $(0,1)$ ), we have

$$
\omega_{a, p}\left(\operatorname{int}_{\partial \Omega} E_{r}\right)=\omega_{a, p}\left(E_{r}\right)=\omega_{a, p}\left(\bar{E}_{r}\right) \quad \text { for all } a \in \Omega .
$$

This result also holds in the weighted Euclidean setting considered in HeinonenKilpeläinen-Martio 9 (since we do not use the invariance of the $p$-harmonic measure under affine transformations in the proof below) or even more generally in the metric space setting considered in Björn-Björn-Shanmugalingam [2] and [3].

Proof. The function $r \mapsto \bar{\omega}_{a, p}\left(\bar{E}_{r}\right)$ is a real-valued nondecreasing function of $r$ and hence differentiable almost everywhere in the interval $(0,1)$. We will only need that it is continuous almost everywhere. Similarly $r \mapsto \underline{\omega}_{a, p}\left(\bar{E}_{r}\right)$ is continuous almost everywhere. Let $A$ be a countable dense subset of $\Omega$ and let $B \subset(0,1)$ be the set of points $r$ such that for every $a \in A$, the functions $\rho \mapsto \bar{\omega}_{a, p}\left(\bar{E}_{\rho}\right)$ and $\rho \mapsto \underline{\omega}_{a, p}\left(\bar{E}_{\rho}\right)$ are continuous at $\rho=r$. Then almost every $r \in(0,1)$ belongs to $B$.

If $0<s<r<1$, then we can find $f \in C(\partial \Omega)$ such that $f=1$ on $\bar{E}_{s}$ and $f=0$ on $\partial \Omega \backslash \operatorname{int}_{\partial \Omega} E_{r}$. It follows that

$$
\bar{\omega}_{a, p}\left(\bar{E}_{s}\right) \leq P f \leq \underline{\omega}_{a, p}\left(\operatorname{int}_{\partial \Omega} E_{r}\right) \text { for all } a \in \Omega .
$$

Therefore for all $a \in A$ and $r \in B$,

$$
\bar{\omega}_{a, p}\left(\bar{E}_{r}\right)=\lim _{s \rightarrow r-} \bar{\omega}_{a, p}\left(\bar{E}_{s}\right) \leq \underline{\omega}_{a, p}\left(\operatorname{int}_{\partial \Omega} E_{r}\right) .
$$

Combining this inequality with the obvious inequalities

$$
\underline{\omega}_{a, p}\left(\operatorname{int}_{\partial \Omega} E_{r}\right) \leq \underline{\omega}_{a, p}\left(E_{r}\right) \leq \underline{\omega}_{a, p}\left(\bar{E}_{r}\right) \leq \bar{\omega}_{a, p}\left(\bar{E}_{r}\right)
$$

and

$$
\underline{\omega}_{a, p}\left(\operatorname{int}_{\partial \Omega} E_{r}\right) \leq \bar{\omega}_{a, p}\left(\operatorname{int}_{\partial \Omega} E_{r}\right) \leq \bar{\omega}_{a, p}\left(E_{r}\right) \leq \bar{\omega}_{a, p}\left(\bar{E}_{r}\right)
$$

we see that

$$
\omega_{a, p}\left(\operatorname{int}_{\partial \Omega} E_{r}\right)=\omega_{a, p}\left(E_{r}\right)=\omega_{a, p}\left(\bar{E}_{r}\right) \quad \text { for } a \in A \text { and } r \in B \text {. }
$$

Thus for $r \in B$ the continuous functions $a \mapsto \omega_{a, p}\left(\operatorname{int}_{\partial \Omega} E_{r}\right), a \mapsto \omega_{a, p}\left(E_{r}\right)$ and $a \mapsto \omega_{a, p}\left(\bar{E}_{r}\right)$ are equal on the dense subset $A$ of $\Omega$, and thus by continuity they are equal on all of $\Omega$. 


\section{The MAIN THEOREM}

The primary aim of this note is to study some conditions on the domain $\Omega$ and the set $E \subset \partial \Omega$ which guarantee that the $p$-harmonic measure of $E$ relative to $\Omega$ satisfies the condition

$$
\omega_{a, p}\left(\operatorname{int}_{\partial \Omega} E\right)=\omega_{a, p}(E)=\omega_{a, p}(\bar{E}) .
$$

The main condition involves a Riesz integral on $\partial \Omega$ which satisfies the integrability hypothesis of the trace theorem of Jonsson-Wallin [10] (see also Hajłasz-Martio 8]).

We denote the $d$-dimensional Hausdorff measure by $\Lambda_{d}$.

Theorem 4.1. Let $1<p<n, \Omega \subset \mathbf{R}^{n}$ be a bounded domain, $E \subset \partial \Omega$, and assume that $C_{p}\left(\partial_{\partial \Omega} E\right)=0$ (which in particular holds if $\Lambda_{n-p}\left(\partial_{\partial \Omega} E\right)<\infty$ ). Moreover, let $U_{1}, \ldots, U_{m} \subset \partial \Omega$ be relatively open sets such that $\partial \Omega=\bigcup_{j=1}^{m} U_{j}$, and for $1 \leq j \leq m$ let $0 \leq d_{1, j} \leq d_{2, j} \leq n$ be such that there is a finite Borel measure $\mu_{j}$ with $\mu_{j}\left(\mathbf{R}^{n} \backslash U_{j}\right)=0$ and

$$
C r^{d_{2, j}} \leq \mu_{j}(B(x, r)) \leq C r^{d_{1, j}} \quad \text { for } x \in U_{j} \text { and } 0<r \leq 1 .
$$

For each $j, 1 \leq j \leq m$, assume that one of the following conditions hold:

(a) $C_{p}\left(U_{j} \cap E\right)=0$ (which in particular holds if $\Lambda_{n-p}\left(U_{j} \cap E\right)<\infty$ );

(b) $C_{p}\left(U_{j} \backslash E\right)=0$ (which in particular holds if $\Lambda_{n-p}\left(U_{j} \backslash E\right)<\infty$ );

(c) $d_{2, j} \leq n-p$;

(d) $d_{2, j}<n$ and

$$
\int_{U_{j} \cap E} \int_{U_{j} \backslash E}|x-y|^{n-p-2 d_{2, j}} d \mu_{j}(y) d \mu_{j}(x)<\infty ;
$$

(e) $d_{2, j}<n$ and

$$
\int_{U_{j} \cap \bar{E}} \int_{U_{j} \backslash \bar{E}}|x-y|^{n-p-2 d_{2, j}} d \mu_{j}(y) d \mu_{j}(x)<\infty ;
$$

(f) $d_{2, j}<n$ and

$$
\int_{U_{j} \cap \operatorname{int}_{\partial \Omega} E} \int_{U_{j} \backslash \operatorname{int}_{\partial \Omega} E}|x-y|^{n-p-2 d_{2, j}} d \mu_{j}(y) d \mu_{j}(x)<\infty ;
$$

(g) $d_{2, j}<n, \operatorname{dim}_{M} U_{j} \cap \partial_{\partial \Omega} E<n-p-2\left(d_{2, j}-d_{1, j}\right)$ and

$$
\sup _{x \in U_{j} \backslash \bar{E}} \frac{\operatorname{dist}\left(x, U_{j} \cap \partial_{\partial \Omega} E\right)}{\operatorname{dist}\left(x, U_{j} \cap E\right)}<\infty ;
$$

(h) $\operatorname{dim}_{M} U_{j} \cap \partial_{\partial \Omega} E<n-p$ and $U_{j}$ is a Lipschitz graph, i.e. there is a convex set $K_{j} \subset \mathbf{R}^{n-1}$, a Lipschitz function $\phi_{j}: \mathbf{R}^{n-1} \rightarrow \mathbf{R}$ and an affine bijection $\Gamma_{j}: \mathbf{R}^{n-1} \times \mathbf{R} \rightarrow \mathbf{R}^{n}$ such that $U_{j}=\left\{\Gamma_{j}\left(x^{\prime}, \phi_{j}\left(x^{\prime}\right)\right): x^{\prime} \in K_{j}\right\}$.

Then $\chi_{E}$ belongs to the trace space of $W^{1, p}\left(\mathbf{R}^{n}\right)$ to $\partial \Omega$ and

$$
\omega_{a, p}\left(\operatorname{int}_{\partial \Omega} E\right)=\omega_{a, p}(E)=\omega_{a, p}(\bar{E}) \quad \text { for all } a \in \Omega \text {. }
$$

Let us start by obtaining Propositions 1.1 and 1.2 as corollaries.

Proof of Proposition 1.2. The boundary is locally as in (h) of Theorem 4.1. That a finite number of sets $U_{j}$ are sufficient follows by compactness. We can therefore apply Theorem 4.1 to complete the proof. 
Proof of Proposition 1.1. This follows directly from Proposition 1.2 for $1<p<2$. For $p=2$ it follows directly from linearity together with the well-known fact that the harmonic measure of a finite set is zero. For $p>2$ (and $m=1$ ) we gave a proof in Section 3 ,

The method of this proof of Proposition 1.1 for $1<p<2$ cannot be extended to cover the case $p>2$. In fact even in the case when $E \subset \partial \mathbf{D}$ is an open arc, $\chi_{E}$ does not belong to the trace space of $W^{1, p}\left(\mathbf{R}^{2}\right)$ when $2 \leq p<\infty$.

Let us next make some comments on Theorem 4.1

The double inequality (4.1) is essential only for the cases (c) (g). Moreover, the upper bound in (4.1) is used only in (g) For the conditions (a) (b) and (h) the measure $\mu_{j}$ is not needed at all. However (4.1) is trivially satisfied if we let $d_{1, j}=0$ and $d_{2, j}=n$, so assuming (4.1) in all cases is no extra assumption. To see this we give the following construction for $\mu_{j}$ : Assume, without loss of generality, that $U_{j} \subset I:=[0,1)^{n}$. We let $\mu_{j}\left(U_{j}\right)=1$. Next divide $I$ into $2^{n}$ cubes similar to $I$ but with half the side length. Say that $a$ of these cubes intersect $U_{j}$; then we let each of these $a$ cubes have measure $1 / a$. In the next step we consider one of these cubes and subdivide it into cubes with half its side length. Say that $b$ of these cubes intersect $U_{j}$; then we let each of these $b$ cubes have the measure $1 / b$ times the measure of the parent cube, i.e. the measure is $1 / a b$. We proceed in this manner to obtain a measure $\mu_{j}$ which satisfies (4.1) with $d_{1, j}=0$ and $d_{2, j}=n$.

In view of (c) conditions (d) (h) are only interesting if $d_{2, j}>n-p$. If (g) is fulfilled with $d_{2, j}>n-p$, then $0<n-p-2\left(d_{2, j}-d_{1, j}\right)$, i.e. $d_{1, j}>d_{2, j}-\frac{1}{2}(n-p)>$ $\frac{1}{2}(n-p)>0$. Since $\Omega$ is a bounded domain, $d_{2, j} \geq n-1$ for at least one $j$.

If $d_{1, j}=d_{2, j}$, then $\mu_{j}$ is equivalent to the Hausdorff measure $\left.\Lambda_{d_{1, j}}\right|_{U_{j}}$; see Theorem 1 on p. 32 of Jonsson-Wallin [10]. In this case $U_{j}$ is Ahlfors $d_{1, j}$-regular (or a $d_{1, j}$-set).

Note that (4.3) is equivalent to

$$
\sup _{x \in U_{j} \cap E} \frac{\operatorname{dist}\left(x, U_{j} \cap \partial_{\partial \Omega} E\right)}{\operatorname{dist}\left(x, U_{j} \backslash \bar{E}\right)}<\infty .
$$

Moreover $(\mathrm{c}) \Rightarrow(\mathrm{a})$ and $(\mathrm{h}) \Rightarrow(\mathrm{g}) \Rightarrow(\mathrm{d})$ See the proof below.

Let us also point out that (d) and (e) are not equivalent. Let, e.g., $1<p<2$, $\Omega=B(0,2) \backslash[0,1] \subset \mathbf{R}^{3}, U=U_{1}=\partial \Omega$ and $\left.\mu\right|_{\partial B(0,2)}=\left.\Lambda_{2}\right|_{\partial B(0,2)}$ and $\left.\mu\right|_{[0,1]}$ be the restriction of the one-dimensional Lebesgue measure. Note that $d_{1,1}=1$ and $d_{2,1}=2$. Further, let $C \subset[0,1]$ be a Cantor set constructed starting with $[0,1]$ so that in the $k$ th step $2^{k}$ open intervals of length $\alpha_{k}$ are removed and the length of each of the $2^{k+1}$ remaining closed intervals equals $l_{k}=\alpha_{k}^{1 / 2}$. Let $E=[0,1] \backslash C$ and observe that $C_{p}\left(\partial_{\partial \Omega} E\right) \leq C_{p}([0,1])=0$. Since $\bar{E}=[0,1]$, (e) holds. Let us turn to (4.2) and start by interchanging the order of integration and letting $y \in C$. Then there exist pairwise disjoint open intervals $I_{k} \subset E$ of length $\alpha_{k}$ with $\operatorname{dist}\left(y, I_{k}\right) \leq l_{k}$. It follows that

$$
\int_{U \cap E}|x-y|^{n-p-2 d_{2,1}} d \mu(x) \geq \sum_{k=0}^{\infty} \alpha_{k}\left(2 l_{k}\right)^{-1-p}=2^{-1-p} \sum_{k=0}^{\infty} l_{k}^{1-p}=\infty .
$$

Since $\mu(C)=1-\sum_{k=0}^{\infty} 2^{k} \alpha_{k}=1-\sum_{k=0}^{\infty} 2^{k} l_{k}^{2} \geq 1-\sum_{k=0}^{\infty} 2^{k} 2^{-2 k-2}=\frac{1}{2}$, (d) does not hold. 
Proof of Theorem 4.1. Let us first observe that if $\Lambda_{n-p}(A)<\infty$, then $C_{p}(A)=0$ by Theorem 2.27 in Heinonen-Kilpeläinen-Martio [9].

By a standard partition of unity argument, we can obtain functions $\eta_{1}, \ldots, \eta_{m} \in$ $\operatorname{Lip}_{c}\left(\mathbf{R}^{n}\right)$, the space of compactly supported Lipschitz functions, such that

$$
\partial \Omega \cap \operatorname{supp} \eta_{j} \subset U_{j} \quad \text { and } \quad \sum_{j=1}^{m} \eta_{j}(y)=1 \text { for } y \in \partial \Omega .
$$

Next, for each $j, 1 \leq j \leq m$, we want to find a $p$-quasicontinuous function $f_{j} \in W^{1, p}\left(\mathbf{R}^{n}\right)$ which equals $\chi_{E}$ on $U_{j}$. Let us postpone this part of the proof for the moment. We proceed by setting

$$
f=\sum_{j=1}^{m} f_{j} \eta_{j}
$$

It is straightforward to see that $f=\chi_{E}$ on $\partial \Omega$ and that $f$ is $p$-quasicontinuous. Moreover since $\eta_{j} \in \operatorname{Lip}_{c}\left(\mathbf{R}^{n}\right)$, it follows that $f_{j} \eta_{j} \in W^{1, p}\left(\mathbf{R}^{n}\right)$ and hence that $f \in W^{1, p}\left(\mathbf{R}^{n}\right)$.

Since $C_{p}\left(\partial_{\partial \Omega} E\right)=0$, the functions $f, f-\chi_{E \cap \partial_{\partial \Omega} E}$ and $f+\chi_{\partial \partial \Omega} \backslash E$ are $p$ quasicontinuous representatives of the same equivalence class in $W^{1, p}\left(\mathbf{R}^{n}\right)$. Hence, by Corollary 5.2 in Björn-Björn-Shanmugalingam [3, we have for $a \in \Omega$,

$$
\begin{aligned}
& \omega_{a, p}(E)=\operatorname{Pf}(a) \quad=H f(a), \\
& \omega_{a, p}\left(\operatorname{int}_{\partial \Omega} E\right)=P\left(f-\chi_{E \cap \partial_{\partial \Omega} E}\right)(a)=H\left(f-\chi_{E \cap \partial_{\partial \Omega} E}\right)(a), \\
& \omega_{a, p}(\bar{E})=P\left(f+\chi_{\partial_{\partial \Omega} E \backslash E}\right)(a)=H\left(f+\chi_{\partial_{\partial \Omega} E \backslash E}\right)(a),
\end{aligned}
$$

where $H g$ is the $p$-harmonic extension of $g \in W^{1, p}\left(\mathbf{R}^{n}\right)$ from $\partial \Omega$ to $\Omega$; see [3]. The operator $H$ is independent of which representative we choose from an equivalence class in $W^{1, p}\left(\mathbf{R}^{n}\right)$, and thus the terms on the right-hand side of the above three identities coincide, which in turn yields the equality of the left-hand side terms.

It remains to find a $p$-quasicontinuous function $f_{j} \in W^{1, p}\left(\mathbf{R}^{n}\right)$ which equals $\chi_{E}$ on $U_{j}$ for each $j, 1 \leq j \leq m$. Here the proof depends on which of the eight conditions holds for $j$ :

(a) We let $f_{j}=\chi_{U_{j} \cap E}$ be a function which is zero $p$-quasieverywhere.

(b) We let $f_{j}=\eta-\chi_{U_{j} \backslash E}$, where $\eta \in \operatorname{Lip}_{c}\left(\mathbf{R}^{n}\right)$ equals one in a neighbourhood of $\bar{\Omega}$.

(c) Let $0<\varepsilon<\frac{1}{2}$ and cover $U_{j}$ with balls $B\left(x_{k}, \varepsilon\right)$ such that $x_{k} \in U_{j}$. If $k \leq l$ and $B\left(x_{k}, \varepsilon\right) \cap B\left(x_{l}, \varepsilon\right) \neq \emptyset$, then $B\left(x_{l}, \varepsilon\right) \subset B\left(x_{k}, 3 \varepsilon\right)$. By taking a subsequence (also denoted $\left\{x_{k}\right\}_{k}$ by abuse of notation) we therefore have disjoint balls $B\left(x_{k}, \varepsilon\right)$ such that $U_{j} \subset \bigcup_{k} B\left(x_{k}, 3 \varepsilon\right)$. It follows, denoting the $d_{2, j}$-dimensional Hausdorff content of $U_{j}$ at scale $\varepsilon$ by $\Lambda_{d_{2, j}}^{\varepsilon}\left(U_{j}\right)$, that

$$
\Lambda_{d_{2, j}}^{3 \varepsilon}\left(U_{j}\right) \leq \sum_{k}(3 \varepsilon)^{d_{2, j}}=C \sum_{k} \varepsilon^{d_{2, j}} \leq C \sum_{k} \mu_{j}\left(B\left(x_{k}, \varepsilon\right)\right) \leq C \mu_{j}\left(U_{j}\right) .
$$

Letting $\varepsilon \rightarrow 0$, this shows that $\Lambda_{d_{2, j}}\left(U_{j}\right) \leq C \mu_{j}\left(U_{j}\right)<\infty$. Therefore $\Lambda_{n-p}\left(U_{j}\right)$ is finite, and thus (a) and (b) are immediately true. (In fact, we have shown that the upper Minkowski content at scale $\varepsilon$ is $\leq C \mu_{j}\left(U_{j}\right)$ and thus that $\operatorname{dim}_{M} U_{j} \leq n-p$.)

(d) We first observe that

$$
C r^{d_{2, j}} \leq \mu_{j}(B(x, r)) \text { for } x \in \bar{U}_{j} \text { and } 0<r \leq 1,
$$

and that (4.2) also holds if $U_{j}$ is replaced by $\bar{U}_{j}$, since $\mu_{j}\left(\mathbf{R}^{n} \backslash U_{j}\right)=0$. 
A function $\mathcal{E} \chi_{E} \in C^{\infty}\left(\mathbf{R}^{n} \backslash \bar{U}_{j}\right)$ is constructed on p. 157 of Jonsson-Wallin [10]. By the trace theorem on p. 182 in $\left[10, \mathcal{E} \chi_{E} \in W^{1, p}\left(\mathbf{R}^{n}\right)\right.$. (We only use the extension part of the trace result, and hence the comment following Theorem 3 on p. 197 in [10] applies here, enabling us to only require the one-sided estimate (4.5).) We let

$$
f_{j}= \begin{cases}\mathcal{E} \chi_{E} & \text { on } \mathbf{R}^{n} \backslash \bar{U}_{j}, \\ \chi_{E} & \text { on } \bar{U}_{j} .\end{cases}
$$

From the contruction on p. 157 in [10, it is easy to see that $f_{j}$ is continuous on $\mathbf{R}^{n} \backslash \partial_{\partial \Omega} E$, and since $C_{p}\left(\partial_{\partial \Omega} E\right)=0$, it follows that $f_{j}$ is $p$-quasicontinuous.

(e) We let

$$
f_{j}= \begin{cases}\mathcal{E} \chi_{\bar{E}} & \text { on } \mathbf{R}^{n} \backslash \bar{U}_{j}, \\ \chi_{\bar{E}} & \text { on } \bar{U}_{j}\end{cases}
$$

and proceed as in $(\mathrm{d})$.

(f) We let

$$
f_{j}= \begin{cases}\mathcal{E} \chi_{\operatorname{int}_{\partial \Omega} E} & \text { on } \mathbf{R}^{n} \backslash \bar{U}_{j} \\ \chi_{\text {int }_{\partial \Omega} E} & \text { on } \bar{U}_{j}\end{cases}
$$

and proceed as in $(\mathrm{d})$.

(g) If $d_{2, j} \leq n-p$, then (c) holds. We therefore assume that $d_{2, j}>n-p$. Our aim is now to show that condition $(\mathrm{d})$ is satisfied as well.

Let us start with some estimates which follow from the condition

$$
\operatorname{dim}_{M} U_{j} \cap \partial_{\partial \Omega} E<d_{3, j}:=n-p-2\left(d_{2, j}-d_{1, j}\right) .
$$

Let

$$
N_{j}(\rho)=\min \left\{k: U_{j} \cap \partial_{\partial \Omega} E \subset \bigcup_{l=1}^{k} B\left(x_{l}, \rho\right) \text { for some } x_{l} \in \mathbf{R}^{n}, l=1, \ldots, k\right\} .
$$

Also, let $\left(x_{l, \rho}\right)_{l=1}^{N_{j}(\rho)}$ be some finite collection of $x_{l}$ for which the minimum is attained. Since $\operatorname{dim}_{M} U_{j} \cap \partial_{\partial \Omega} E<d_{3, j}$ there is $\varepsilon>0$ such that

$$
N_{j}(\rho) \leq \frac{C}{\rho^{d_{3, j}-\varepsilon}} .
$$

As

$$
\left\{x \in U_{j}: \operatorname{dist}\left(x, U_{j} \cap \partial_{\partial \Omega} E\right)<\rho\right\} \subset \bigcup_{l=1}^{N_{j}(\rho)} B\left(x_{l, \rho}, 2 \rho\right)
$$

and $\mu_{j}\left(B\left(x_{l, \rho}, 2 \rho\right)\right) \leq C \rho^{d_{1, j}}$ (this estimate holds also if $x_{l, \rho} \notin U_{j}$ or $\rho>1$, though the constant $C$ may not be the same as in the statement of the theorem), it follows that

$$
\mu_{j}\left(\left\{x \in U_{j}: \operatorname{dist}\left(x, U_{j} \cap \partial_{\partial \Omega} E\right)<\rho\right\}\right) \leq C N_{j}(\rho) \rho^{d_{1, j}} \leq C \rho^{d_{1, j}-d_{3, j}+\varepsilon} .
$$

Hence, using (4.4), we obtain the estimate

$$
\mu_{j}\left(\left\{x \in U_{j} \cap E: \operatorname{dist}\left(x, U_{j} \backslash \bar{E}\right)<\rho\right\}\right) \leq C \rho^{d_{1, j}-d_{3, j}+\varepsilon} .
$$

We want to prove that condition (d) holds, i.e. that

$$
I:=\int_{U_{j} \cap E} \int_{U_{j} \backslash E}|x-y|^{n-p-2 d_{2, j}} d \mu_{j}(y) d \mu_{j}(x)<\infty .
$$


Let us first estimate the inner integral, fixing $x \in U_{j} \cap \operatorname{int}_{\partial \Omega} E$. (Note that $\mu_{j}\left(\partial_{\partial \Omega} E\right)=0$ by (4.6) .) Let $\delta=\delta(x)=\operatorname{dist}\left(x, U_{j} \backslash E\right)$. Using $n-p-2 d_{2, j}+d_{1, j} \leq$ $n-p-d_{2, j}<0$, we then find that

$$
\begin{aligned}
\int_{U_{j} \backslash E}|x-y|^{n-p-2 d_{2, j}} d \mu_{j}(y) & \leq \int_{U_{j} \backslash B(x, \delta)}|x-y|^{n-p-2 d_{2, j}} d \mu_{j}(y) \\
& \leq C \sum_{j=0}^{\infty}\left(2^{j} \delta\right)^{n-p-2 d_{2, j}} \mu_{j}\left(B\left(x, 2^{j+1} \delta\right)\right) \\
& \leq C \sum_{j=0}^{\infty}\left(2^{j} \delta\right)^{n-p-2 d_{2, j}}\left(2^{j+1} \delta\right)^{d_{1, j}} \\
& \leq C \delta^{n-p-2 d_{2, j}+d_{1, j}}=C \delta^{d_{3, j}-d_{1, j}} .
\end{aligned}
$$

Inserting the above estimate into $I$ and using $\mu_{j}\left(\partial_{\partial \Omega} E\right)=0$ and (4.7) yields

$$
\begin{aligned}
I & \leq C \int_{U_{j} \cap \operatorname{int} \partial \Omega} E(x)^{d_{3, j}-d_{1, j}} d \mu_{j}(x) \\
& \leq C \int_{0}^{\operatorname{diam} \partial \Omega} t^{d_{3, j}-d_{1, j}} \mu_{j}\left(\left\{x \in U_{j} \cap E: \delta(x)<t\right\}\right) \frac{d t}{t} \\
& \leq C \int_{0}^{\operatorname{diam} \partial \Omega} t^{d_{3, j}-d_{1, j}} t^{d_{1, j}-d_{3, j}+\varepsilon} \frac{d t}{t}<\infty .
\end{aligned}
$$

(h) We show that under this condition the set $U_{j}$ satisfies condition (g) as well. Let $M$ be such that $\left|\Gamma_{j}\left(x^{\prime}, \phi_{j}\left(x^{\prime}\right)\right)-\Gamma_{j}\left(y^{\prime}, \phi_{j}\left(y^{\prime}\right)\right)\right| \leq M\left|x^{\prime}-y^{\prime}\right|$ for $x^{\prime}, y^{\prime} \in K_{j}$. We want to show that

$$
\sup _{x \in U_{j} \backslash \bar{E}} \frac{\operatorname{dist}\left(x, U_{j} \cap \partial_{\partial \Omega} E\right)}{\operatorname{dist}\left(x, U_{j} \cap E\right)}<\infty .
$$

Let $x \in U_{j} \backslash \bar{E}$ and $y \in U_{j} \cap E$ be such that $|x-y| \leq 2 \operatorname{dist}\left(x, U_{j} \cap E\right)$. We further let $x^{\prime}, y^{\prime} \in K_{j}$ be such that $x=\Gamma_{j}\left(x^{\prime}, \phi_{j}\left(x^{\prime}\right)\right)$ and $y=\Gamma_{j}\left(y^{\prime}, \phi_{j}\left(y^{\prime}\right)\right)$. Then there exists a minimal $\theta \in(0,1]$ such that if $z^{\prime}=(1-\theta) x^{\prime}+\theta y^{\prime}$, then $z:=\Gamma_{j}\left(z^{\prime}, \phi_{j}\left(z^{\prime}\right)\right) \in \bar{E}$. It follows from the minimality that $z \in \partial_{\partial \Omega} E$. We find by the Lipschitz property of $\varphi_{j}$ that

$$
|x-z| \leq M\left|x^{\prime}-z^{\prime}\right|=M \theta\left|x^{\prime}-y^{\prime}\right| \leq C|x-y|
$$

(where $C$ depends only $M$ and the stretching of $\Gamma_{j}$ ), and thus

$$
\frac{\operatorname{dist}\left(x, U_{j} \cap \partial_{\partial \Omega} E\right)}{\operatorname{dist}\left(x, U_{j} \cap E\right)} \leq 2 \frac{|x-z|}{|x-y|} \leq C .
$$

This shows that condition (g) is fulfilled with the measure $\mu_{j}=\left.\Lambda_{n-1}\right|_{U_{j}}$ and $d_{1, j}=d_{2, j}=n-1$; see, e.g., Example 3 on p. 30 in Jonsson-Wallin [10] to see that $\mu_{j}$ satisfies our conditions.

\section{REFERENCES}

[1] A. Baernstein II, Comparison of p-harmonic measures of subsets of the unit circle, St. Petersburg Math. J. 9 (1998), 543-551. MR1466798 (2000e:30043)

[2] A. Björn, J. Björn, and N. Shanmugalingam, The Dirichlet problem for p-harmonic functions on metric spaces, J. Reine Angew. Math. 556 (2003), 173-203. MR 1971145 (2004e:31007)

[3] _ The Perron method for p-harmonic functions in metric spaces, J. Differential Equations 195 (2003), 398-429. MR2016818 (2004i:31012) 
[4] Sobolev extensions of Hölder continuous and characteristic functions on metric spaces, in preparation.

[5] D. Danielli, N. Garofalo, and D. M. Nhieu, Sub-elliptic Besov spaces and the characterization of traces on lower dimensional manifolds, in Harmonic Analysis and Boundary Value Problems (Fayetteville, Ark., 2000), Contemp. Math. 277, pp. 19-37, Amer. Math. Soc., Providence, R.I., 2001. MR.1840425 (2002f:46049)

[6] _ Non-doubling Ahlfors Measures, Perimeter Measures, and the Characterization of the Trace Spaces of Sobolev Functions in Carnot-Carathéodory Spaces, to appear in Mem. Amer. Math. Soc., Amer. Math. Soc., Providence, R.I.

[7] H. Federer, Geometric Measure Theory, Springer-Verlag, Berlin-Heidelberg, 1969. MR0257325 (41:1976)

[8] P. Hajłasz and O. Martio, Traces of Sobolev functions on fractal type sets and characterization of extension domains, J. Funct. Anal. 143 (1997), 221-246. MR.1428124 (98d:46034)

[9] J. Heinonen, T. Kilpeläinen, and O. Martio, Nonlinear Potential Theory of Degenerate Elliptic Equations, Oxford Univ. Press, Oxford, 1993. MR1207810 (94e:31003)

[10] A. Jonsson and H. Wallin, Function Spaces on Subsets of $\mathbf{R}^{n}$, Math. Rep. 2:1, Harwood, London, 1984. MR0820626 (87f:46056)

[11] J. Kurki, Invariant sets for A-harmonic measure, Ann. Acad. Sci. Fenn. Ser. A I Math. 20 (1995), 433-436. MR1346825 (97g:31013)

[12] J. J. Manfredi, p-harmonic functions in the plane, Proc. Amer. Math. Soc. 103 (1988), 473-479. MR0943069 (89f:35076)

[13] P. Mattila, Geometry of Sets and Measures in Euclidean Spaces, Cambridge Univ. Press, Cambridge, 1995. MR.1333890 (96h:28006)

[14] W. P. Ziemer, Weakly Differentiable Functions, Springer-Verlag, New York, 1989. MR:1014685 (91e:46046)

Department of Mathematics, Linköpings Universitet, SE-581 83 Linköping, Sweden

E-mail address: anbjo@mai.liu.se

Department of Mathematics, Linköpings Universitet, SE-581 83 Linköping, Sweden

E-mail address: jabjo@mai.liu.se

Department of Mathematical Sciences, University of Cincinnati, P.O. Box 210025, Cincinnati, Ohio 45221-0025

E-mail address: nages@math.uc.edu 